\title{
Le développement des cornets nasaux chez le Porc, son appréciation, aspects génétiques
}

\author{
par D. PLANCHENAULT *, P. SELLIER, L. OLLIVIER
}

avec la collaboration technique de Marie-Reine LANGLOIS, P. DANDO, C. FELGINES et J. GRUAND

Déparfement de Génétique animale, I.N.R.A.

78350 Jouy-en-Josas.

Summary. Assessment of nasal turbinate development in the pig. Genetic aspects.

Between 1972 and 1975 we studied 4236 pigs, mainly of the Large White breed, from two experimental farms of the I.N.R.A. Their snouts were radiographed between 20 and $30 \mathrm{~kg}$ liveweight, and 2028 of them, when reaching $100 \mathrm{~kg}$ liveweight, were further examined for turbinate atrophy by a transversal section of the snout. Radiographs were scored from 0 to 5 , according to the degree of atrophy and snout sections were similarly scored from 0 to 4 . There was a highly significant correlation of 0.37 between the two scores on the same animal. The percentage of animals showing no sign of nasal alteration at slaughter was the same as the percentage obtained by radiography, thus indicating no marked increase in turbinate atrophy of the total population over the fattening period. However, among pigs with no nasal alterations at radiography, 5.6 p. 100 showed severe alterations of the snout section which corresponded to clinical signs of atrophic rhinitis. Male and female radiographs did not significantly differ, whereas, as judged from snout section at slaughter, males were more severely affected than females. The average scores of entire and castrated males were higher by 0.28 and 0.18 , respectively, than those of females. On the average, heritabilities estimated from half-sib correlations were higher for radiograph score (0.09 and 0.21 in the two farms) than for snout section score $(0.10$ and 0.03 in the two farms). Parent-offspring regression on radiographs yielded lower estimates than the previous method; this might be due to non-linearity of regression and selection of parents.

\section{Introduction.}

Les fosses nasales, chez le Porc, ont la particularité de porter sur leurs parois externes deux cornets, de chaque côté de la fête (Jost, 1973). Cette structure particulièrement fragile est d'un accès difficile parce que presque complètement entourée d'os. Au cours de la croissance, l'os est soumis à un renouvellement intense et il se trouve que le métabolisme osseux des cornets nasaux est lui-même très particulier, ef caractérisé par un renouvellement plus rapide que celui des autres os (Besançon, 1972). Cela rend ces cornets très sensibles à des désordres métaboliques d'origine

* Cet article a fait, en partie, l'objet d'un mémoire de D.E.A. en génétique quantitative, présenté à l'Université de Paris VI. 


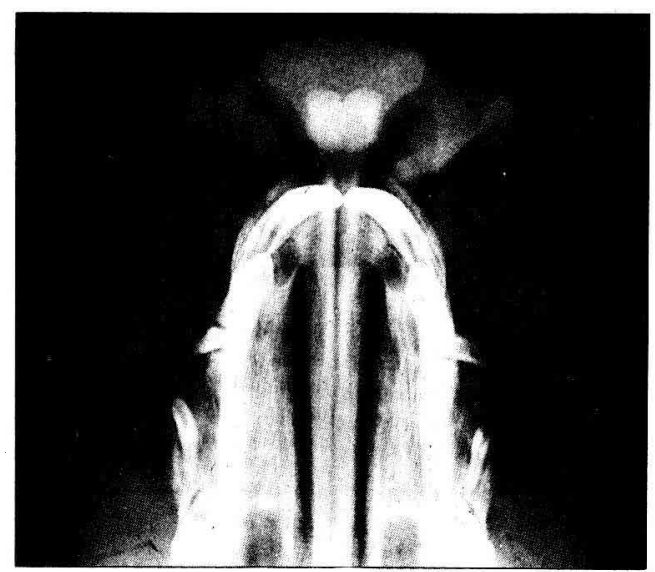

Radiographie a

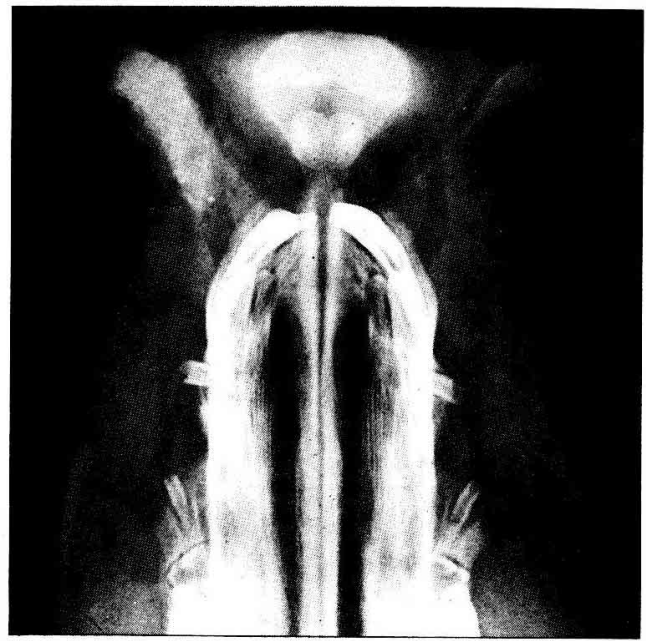

Radiographie c

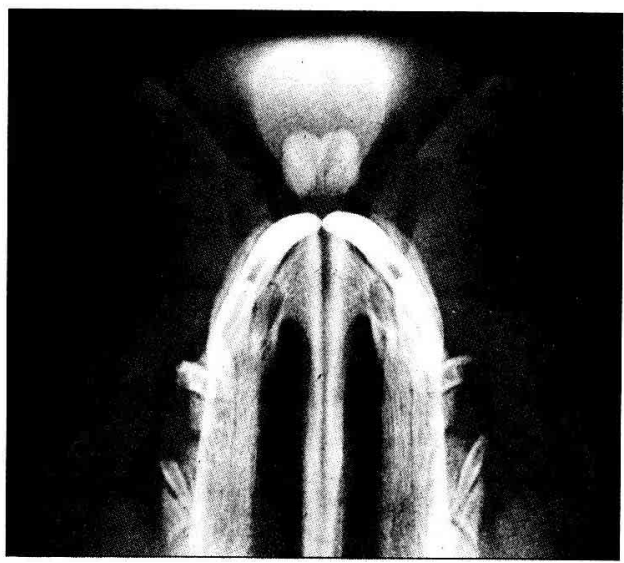

Radiographie e

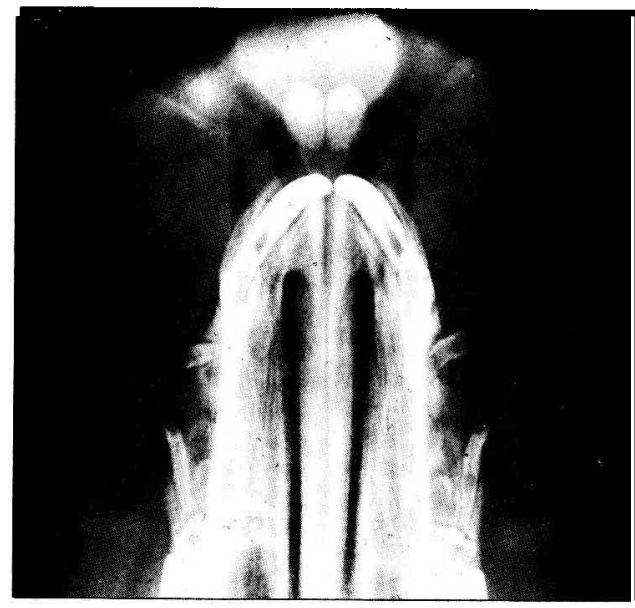

Radiographie b

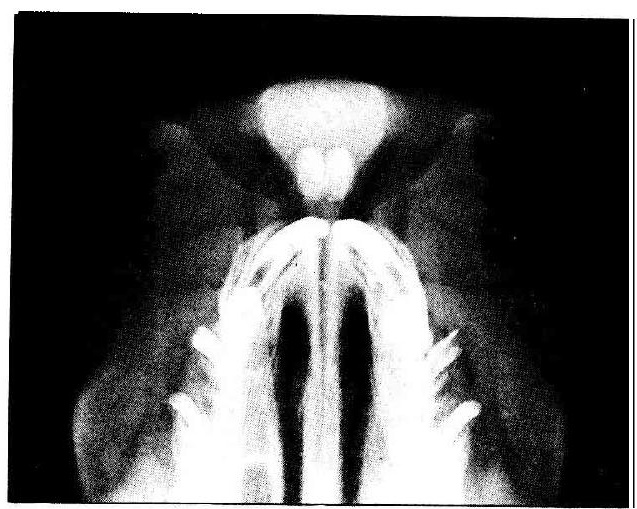

Radiographie d

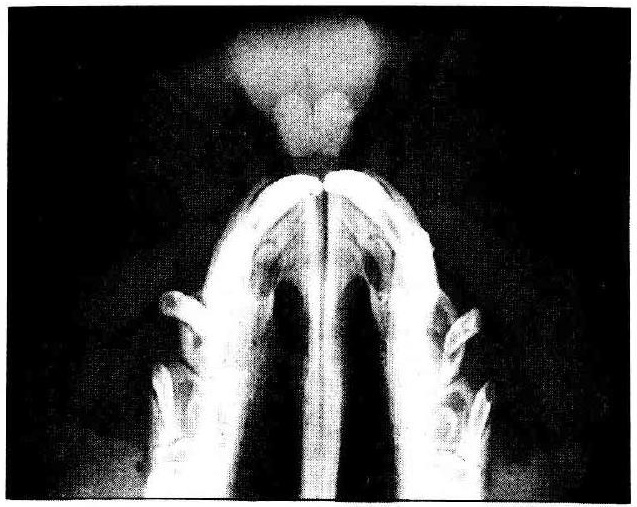

Radiographie $f$

FIG. 1. - Notation radiogrophique : les clichés $a, b, c, d$, e, f correspondent respectivement aux notes $0,1,2,3,4,5$. 
diverse qui peuvent engendrer ce qu'on a appelé le syndrome de la rhinite atrophique, dont les causes sont probablement multiples. Nous prendrons ici le terme de rhinite atrophique dans un sens large d'inflammation aiguë ou chronique de la muqueuse pituitaire avec une altération plus ou moins marquée des cornets nasaux sans référence à une éfiologie exacte, ni à une forme anatomopathologique précise. Cette maladie, peu spécifique, a une longue histoire et a donné lieu à des méthodes de diagnostic nombreuses et variées (Planchenault, 1976). Dans cette étude, deux de ces méthodes ont été retenues, la radiographie de la mâchoire supérieure de l'animal au début de l'engraissement (entre 20 et $30 \mathrm{~kg}$ ) ef la coupe transversale du nez à l'abattage (vers $100 \mathrm{~kg}$ ). Il est ainsi possible de suivre sur chaque individu l'évolution de l'état des cornets nasaux entre le début et la fin de l'engraissement. L'analyse qui va être présentée a deux objectifs : déterminer le degré de concordance entre ces deux techniques et mettre en évidence d'éventuels facteurs héréditaires prédisposant aux altérations des cornets nasaux.

\section{Matériel et méthodes.}

En 1972, l'INRA a mis en place une expérimentation en vue d'étudier l'aspect génétique des altérations nasales chez le Porc, dans deux élevages expérimentaux du Département de Génétique animale, situés l'un à Avord (Cher) l'autre à Rouillé (Vienne). Tous les porcs entrant en engraissement sont radiographiés ef les clichés sont examinés et classés selon une échelle décrite par Dando et al. (1973), qui va de 0 à 5 selon le degré d'altération des cornets (fig. 1). A l'abattage, vers $100 \mathrm{~kg}$, les nez sont coupés à la scie (fig. 2), examinés et classés selon le barème décrit par les auteurs précédents, la note donnée allant de 0 à 4 selon le degré d'atrophie observé. L'échantillon analysé comprend 4236 porcs pour l'examen radiographique el 2028 pour la coupe du nez, en majorité de race Large White (3198 et 1506 respectivement pour les radiographies et les coupes), le reste étant réparti en Piétrain, Piétrain $\times$ Large

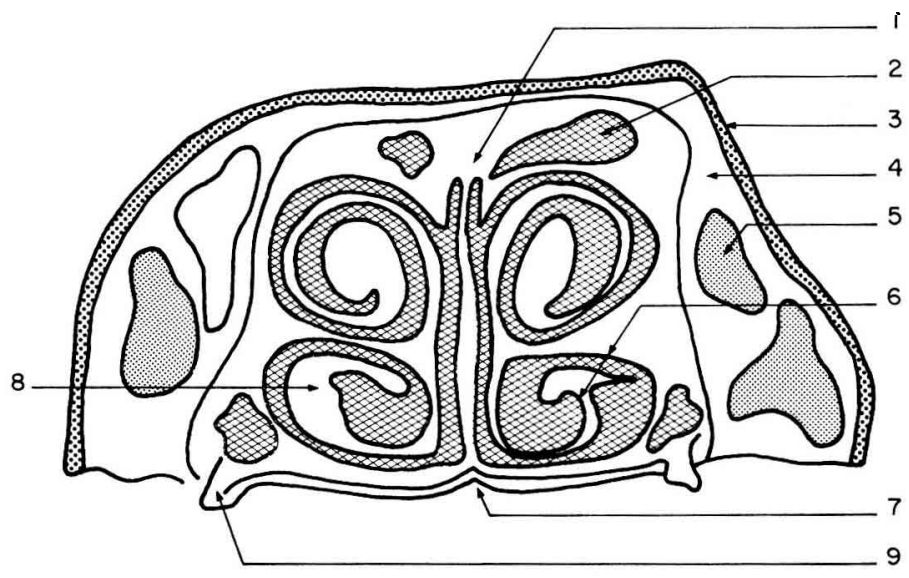

FIG. 2. - Schéma d'une coupe transversale de groin de Porc

1. Cloison médiane ; 2. Sinus ; 3. Tissu cutané ; 4. Tissu conjonctivo-adipeux ; 5 . Tissu musculaire ; 6. Fosses nasales ; 7. Vomer ; 8. Cornets nasaux ; 9. $1^{\text {re }}$ prémolaire. 
White, Hampshire $\times$ Large White ef Piétrain $\times$ Hampshire $\times$ Large White. Tous ces porcs ont été engraissés en bâtiments de semi-plein air, par loge de 10 , et alimentés à volonté au nourrisseur.

L'analyse statistique comporte une première phase d'estimation des effets non génétiques, à savoir le sexe, la bande d'engraissement ef l'interaction sexe $x$ bande. Pour la note radiographique, les mâles entiers et castrés sont regroupés, car le faible intervalle qui sépare la castration de l'examen radiographique inciłe à penser que l'opération est sans effet sur l'état des cornets. Pour les notes de coupe, seules sont retenues pour l'analyse les bandes où tous les mâles sont castrés, puisque à Avord le choix des mâles à castrer est lié au résultat de l'examen radiographique, et les mâles entiers ne sont pas soumis à la coupe.

La deuxième phase de l'analyse consiste à estimer les effets génétiques. Deux méthodes de calcul sont utilisées. La première est basée sur la régression parentdescendant, qui s'applique aux données radiographiques seulement, puisque la coupe nécessite l'abattage. L'échantillon d'Avord permet d'estimer une régression parent moyen-descendant, puisque les pères et les mères ont subi l'examen radiographique. Par contre, à Rouillé, seuls les pères ont été examinés et la régression pondérée père-descendant est alors calculée, selon la méthode décrite par Ollivier (1974). La deuxième méthode est basée sur l'analyse de variance, selon un modèle « mixte » incluant deux facteurs fixés, bande et sexe, et trois facteurs aléatoires constituant une hiérarchie père-mère-descendant. Dans cette analyse, qui concerne à la fois les notes radiographiques et les notes de coupe, les données de chaque élevage ont été traitées séparément. Cela a permis, sur les notes de coupe de Rouillé, de comparer les femelles, les mâles entiers et les mâles castrés, ce qui n'était pas possible dans l'échantillon d'Avord. Selon la méthode employée, l'héritabilité a été estimée comme égale soit à la régression parent moyen-descendant, soit au double de la régression père-descendant, soit enfin à 4 fois la corrélation entre demi-frères obtenue dans l'analyse de variance avec modèle mixte.

\section{Résultats.}

Le tableau 1 permet de situer globalement les 2 élevages étudiés l'un par rapport à l'autre. Les cornets nasaux sont en moyenne plus atteints à Avord qu'à Rouillé, que

TABLEAU 1

Moyenne ef écart-type des échantillons

\begin{tabular}{|c|c|c|c|c|}
\hline & & Avord & Rouillé & Total \\
\hline Radio & $\begin{array}{l}\text { Effectif .......... } \\
\text { Moyenne ........ } \\
\text { Ecart-type ....... }\end{array}$ & $\begin{array}{r}2530 \\
2,35 \\
1,11\end{array}$ & $\begin{array}{r}1706 \\
1,16 \\
0,80\end{array}$ & $\begin{array}{l}4236 \\
1,83\end{array}$ \\
\hline Coupe & $\begin{array}{l}\text { Effectif } \ldots \ldots \ldots \ldots \\
\text { Moyenne ....... } \\
\text { Ecart-type ....... }\end{array}$ & $\begin{array}{r}522 \\
1,55 \\
1,07\end{array}$ & $\begin{array}{r}1506 \\
1,21 \\
0,90\end{array}$ & $\begin{array}{l}2028 \\
1,43\end{array}$ \\
\hline
\end{tabular}


ce soit au début de l'engraissement (note de radio) ou à l'abattage (note de coupe) Notons cependant que la différence appréciée par radiographie, supérieure à un écart-type sur l'échelle de notation, est nettement plus importante que celle jugée sur la coupe qui est de l'ordre d'un tiers d'écart-type.

La figure 3 donne les distributions des 2 variables étudiées sur les échantillons globaux, et révèle une dissymétrie marquée des 2 distributions. L'examen des distributions par élevage montre que la dissymétrie affecte surtout les données de Rouillé.
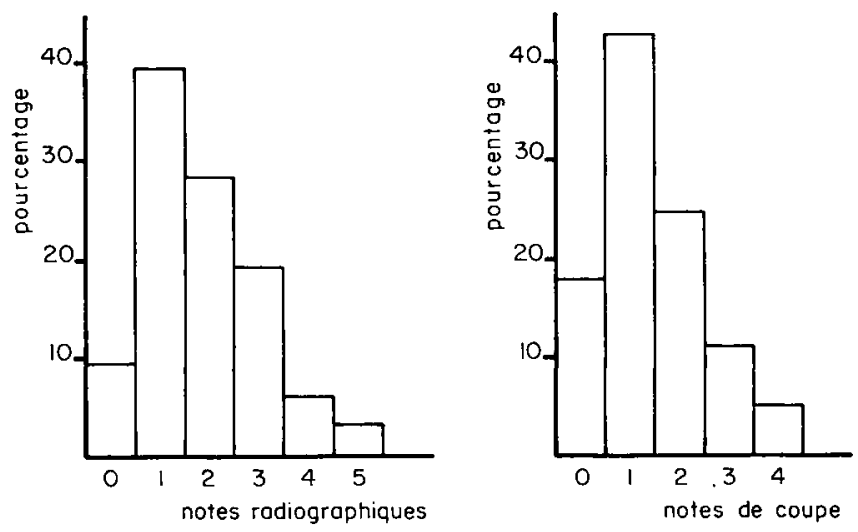

FIG. 3. - Distributions des notes radiographiques ef des notes de coupe sur l'ensemble de l'échantillon.

La relation entre l'examen radiographique en début d'engraissement et l'examen du nez à l'abattoir sur le même porc est illustrée par la figure 4. La corrélation entre les deux notes est de 0,37 . Remarquons cependant que la relation n'est visiblement pas linéaire et que, de plus, la variabilité de la notation des coupes tend à augmenter avec le degré d'altération des cornets tel qu'il est apprécié par radiographie. Cela rend la corrélation précédente peu adéquate pour traduire la relation observée.

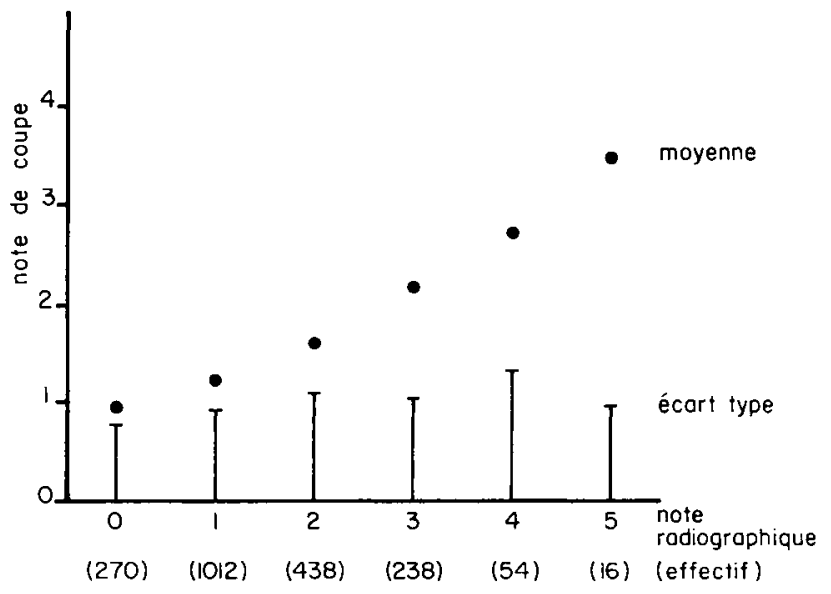

FIG. 4. - Relation entre la notation radiographique et la notation de la ccupe. 
Le tableau 2 donne le résultat des analyses de variance. Une interaction hautement significative sexe $\times$ bande pour les notes radiographiques empêche en toute rigueur de tester les effets principaux dans l'absolu. Les valeurs de F montrent cependant que la bande est un facteur de variation prépondérant et que le sexe a peu d'effet. Par contre à l'abattage, l'effet « bande », très hautement significatif, et l'effet « sexe », hautement significatif, peuvent être considérés comme additifs. Les mâles castrés présentent des cornets en moyenne plus altérés que les femelles, la différence étant de $0,14 \pm 0,01$ point. Sur l'échantillon de Rouillé, les moyennes estimées sonł respectivement $1,11 \pm 0,05,1,29 \pm 0,07$ et $1,39 \pm 0,07$ pour les femelles, les mâles castrés et les mâles entiers.

TABLEAU 2

Analyses de variance

\begin{tabular}{|c|c|c|c|c|}
\hline \multirow{2}{*}{ Cause de variation } & \multicolumn{2}{|c|}{ Degrés de liberté } & \multicolumn{2}{|c|}{ Carrés moyens } \\
\hline & Radio & Coupe & Radio & Coupe \\
\hline $\begin{array}{l}\text { Bande } \ldots \ldots \ldots \ldots \ldots \ldots \ldots \ldots \\
\text { Sexe } \ldots \ldots \ldots \ldots \ldots \\
\text { Interaction bande } \times \text { sexe } \ldots \ldots \ldots \\
\text { Résiduelle } \ldots \ldots \ldots \ldots \ldots \ldots\end{array}$ & $\begin{array}{r}11 \\
1 \\
11 \\
3743\end{array}$ & $\begin{array}{r}5 \\
1 \\
5 \\
1370\end{array}$ & $\begin{array}{c}120,56 \\
1,29 \\
2,77 * * \\
1,05\end{array}$ & $\begin{array}{l}35,98 * * * \\
6,53 * * \\
1,70 \mathrm{NS} \\
0,96\end{array}$ \\
\hline
\end{tabular}

NS : Test $F$ non significatif.

**: Test $\mathrm{F}$ significatif au seuil de 1 p. 100.

*** : Test $F$ significatif au seuil de 1 p. 1000.

Les estimations d'héritabilité du tableau 3 font ressortir que, sur les mêmes données, les valeurs obtenues par régression sont moins élevées que celles obtenues par l'analyse de variance. De plus, les résultats de l'analyse de variance indiquent que les facteurs héréditaires semblent avoir moins d'influence sur l'état des cornets apprécié sur la coupe à l'abattage que sur le degré des altérations observées par radiographie en début d'engraissement.

TABLEAU 3

Héritabilités

\begin{tabular}{|c|c|c|c|}
\hline \multirow{2}{*}{ Méthode d'estimation } & \multirow{2}{*}{ Variable } & \multicolumn{2}{|c|}{ Elevage } \\
\hline & & Avord & Rouillé \\
\hline Régression parent-descendant............ & Radio & $-0,07 \pm 0,11$ & $0,14 \pm 0,08$ \\
\hline $\begin{array}{l}\text { Analyse de variance }(4 \times \text { corrélation entre } \\
\text { demi-frères }) \ldots \ldots \ldots \ldots \ldots \ldots \ldots \ldots \ldots \ldots \ldots\end{array}$ & $\begin{array}{l}\text { Radio } \\
\text { Coupe }\end{array}$ & $\begin{array}{l}0,09 \pm 0,03 \\
0,10 \pm 0,08\end{array}$ & $\begin{array}{l}0,21 \pm 0,07 \\
0,03 \pm 0,05\end{array}$ \\
\hline
\end{tabular}




\section{Discussion et conclusions.}

1. Concordance entre les 2 méthodes d'appréciation des cornets nasaux.

L'application à grande échelle des 2 méthodes aux mêmes porcs, au même stade ou à des stades différents, ne semble avoir été réalisée qu'en France, par l'INRA et par l'Institut technique du Porc (entre 1972 et 1975, 15369 jeunes verrats radiographiés), dont les premiers résultats ont été rapportés par Jost (1973). Les deux techniques sont assez grossières et leurs imperfections ont été soulignées par Dando ef al. (1973), Juhl (1974) et Done (1976). Elles donnent cependant, en pourcentage d'altérations détectées, et contrairement à ce que rapporte Done, des résultats comparables, qu'elles soient appliquées au même stade (Jost, 1973) ou, comme ici, à des stades différents : 40 p. 100 de notes de coupe 2,3 et 4 comparés à 37 p. 100 de notes de radio 2 , 3,4 et 5 (figure 4). Notons par ailleurs que les signes cliniques de la rhinite correspondent aux notes de coupe 3 et 4 et que 5, 6 p. 100 des porcs jugés indemnes à l'examen radiographique ( 0 ou 1 ) tombent dans cette catégorie en fin d'engraissement contre 15,9 p. 100 dans l'échantillon fotal. Cela montre l'intérêt pratique et les limites du diagnostic radiographique de la rhinite.

\section{Evolution de l'état des cornets nasaux pendant l'engraissement.}

Cette étude ne peut donner que des indications imparfaites sur cette évolution, puisque 2 techniques différentes sont employées en début et fin d'engraissement. Sur un échantillon de 270 porcs radiographiés à 35 et à $100 \mathrm{~kg}$ par I'I.T.P. le pourcentage d'animaux atteints passait de 19 à 39 p. 100 (données non publiées). Ce résultat indique qu'une aggravation des altérations nasales se produit au cours de l'engraissement, aggravation qui n'apparaît pas à l'examen nécropsique (voir plus haut). Cela explique sans doute que les différences entre les 2 élevages étudiés ici semblent moins marquées sur les notes de coupe que sur les notes de radio, bien qu'on ne puisse exclure des différences d'appréciation des clichés et des coupes entre les 2 notateurs.

L'influence du sexe se traduit, au contraire, par une aggravation décelable à la coupe. Les faibles différences relevées à la radiographie confirment les résultats de Seifert ef al. (1971). Par contre, les différences que nous trouvons à l'abattage n'ont pas été signalées par d'autres auteurs. Jonsson (1965), par exemple, signale des différences faibles et de signe contraire des nôtres entre mâles castrés et femelles. Il est à noter que l'ordre de gravité des altérations correspond au classement sur la vitesse de croissance, c'est-à-dire femelles-mâles castrés-mâles entiers (Desmoulin, 1973 ; Hansson, 1974). Comme Bjorklund (1958) a montré que l'os nasal du porc croît d'une façon continue bien au-delà du poids de $100 \mathrm{~kg}$, on peut penser que les mâles entiers, qui ont la croissance la plus rapide, subissent de ce fait une dégradation plus marquée de leurs cornets nasaux que les mâles castrés et les femelles.

\section{Aspects génétiques.}

Les résultats du tableau 3 ne confirment pas totalement ceux de Jonsson (1965), Seifert ef al. (1971) ef Dando ef al. (1973) quant à l'influence de facteurs génétiques sur le degré des altérations nasales, surtout s'il est apprécié par l'examen nécropsique. 
Par ailleurs, les faibles valeurs d'héritabilité trouvées par régression comparativement à l'analyse de variance pourraient s'expliquer par le fait que, dans notre échantillon, les parents étaient sélectionnés (une note supérieure à 2 entraînant généralement l'élimination), si on suppose que la régression parent-descendant n'est pas linéaire.

De l'ensemble de ces résultats il ressort que l'état des cornets nasaux à l'abattage est, dans une large mesure, le résultat d'une dégradation intervenant précocement et dépendant partiellement du patrimoine héréditaire, dont l'influence s'exerce peutêtre à travers le potentiel de croissance de l'individu, comme les différences entre sexes pourraient l'indiquer.

Réunion Groupe Développement INRA/Productions animales Montpellier, 17-18 mai 1977.

\section{Références}

BESANÇON P., 1972. Aspects nutritionnels et métaboliques liés à la rhinite atrophique, 13-27. In la Rhinife atrophique du Porc. Instifuł technique du Porc, Paris.

BJORKLUND N. E., 1958. Atrophic rhinitis of pigs. Thèse vétérinaire, Stockholm PP. 100.

DANDO P., GRUAND J., OLLIVIER L., 1973. Examens radiographiques ef nécropsiques de nez de porc : premiers résultats, 23-31. In Journées Rech. Porcine en France 1973. Institut technique du Porc, Paris.

DESMOULIN B., 1973. Qualités de carcasse des porcs Large White, aptitudes aux rationnements suivant le sexe et après la castration, 189-199. In Journées Rech. Porcine en France 1973. Institut technique du Porc, Paris.

DONE J. T., 1976. Porcine atrophic rhinitis. Snout radiography as and aid to diagnosis and detection of the disease. Vet. Rec., 98, 23-28.

HANSSON I., 1974. Effect of sex and weight on growth, feed efficiency and carcass characteristics of pigs. I. Growth rate and feed efficiency of boars, barrows and gilts. Swed. J. agric. Res., 4, 209-218.

JONSSON P., 1965. Analyse des caractères du porc Landrace danois avec une introduction historique (en Danois). 350. Beret. forsøgslabor., 490 pp.

JOST P., 1973. La rhinite atrophique du Porc. Dépistage et prophylaxie. Recl. Méd. vét. Ec. Alfort, 149, 997-1020.

JUHL K., 1974. Projektions-und Interpretationsfehler bei der Rönfgenanatomischen Darstellung der Nasenhöhle des Schweines. Diss. Tierärztl. Hochschule, Hannover.

OLLIVIER L., 1974. La régression parent-descendant dans le cas de descendances subdivisées en familles de taille inégale. Biometrics, 30, 59-66.

PLANCHENAULT D., 1976. Aspects génétiques de la rhinite atrophique du porc. Mémoire de DEA. Univ. Paris Vl, 53 pp.

SEIFERT H., SCHÖNMUTH G., NAGEL E., 1971. Ergebnisse experimenteller Untersuchungen auf Wirkung genetischer Faktoren bei der Rhinitis atrophicans suum. Wiss. Zeit. Humboldt Univ. Berlin, 20, 371-387. 